\title{
Multiple renal arteries - a pitfall in renovascular intervention
}

\section{Basil J Sher FCRad(D)SA \\ Ian C Duncan FFRad(D)SA \\ Sunninghill Medical Institute Sandton}

\section{Case report}

A 51-year-old man presented 7 days after a stab wound of the lower left chest posteriorly with continuous macroscopic haematuria. He was referred to our unit for arteriographic assessment of the kidneys and immediate endovascular management of any appropriate vascular injury thus discovered. An abdominal CT scan allegedly showed a major renal injury with a perinephric haematoma. This scan was unfortunately not available to us for personal review at the time of the angiogram.

Upon admission to the angiography suite the patient was stable haemodynamically. Obvious macroscopic haematuria was seen in the urinary catheter drainage bag. An initial abdominal aortic flush run was performed. This showed a normal intact right kidney and no obvious abnormality of the left kidney but did indicate the presence of multiple left renal arteries (Fig. 1). The exact number of these could not be assessed and the left renal nephrogram was poorly visualised mainly due to image degradation due to cardiac, respiratory and bowel motion artefact. Selective catheterisation of the left renal arteries was then undertaken using a $4 \mathrm{~F} \mathrm{C} 2$ catheter over a hydrophilic guidewire. The first renal artery catheterised sup-

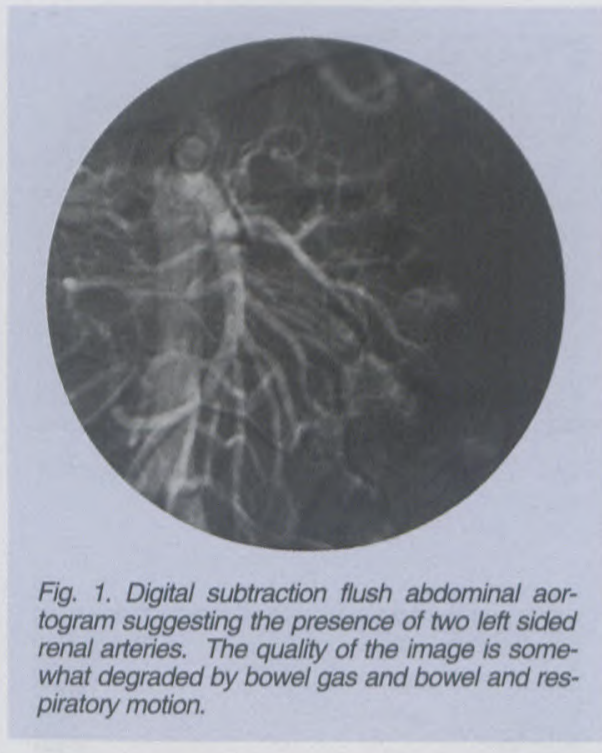

plied the lower pole and other than mild catheter-induced spasm appeared normal (Fig. 2A). The second artery catheterised supplied areas of the middle and upper pole regions of the kidney (Fig. 2B). An irregularly contoured parenchymal outline was seen which was initially considered to be due to the perinephric haematoma. The angiogram had not revealed any visible intrarenal vascular injury at this stage, and it was decided at this point to stop the procedure. However, after further analysis of the available images it was decided that the summated nephrographic pattern was incomplete and that further attempts be made to try and identify other left renal branches that might reveal a significant vascular injury. A third renal artery was thus eventually found. Selective angiography of this vessel showed filling of a pseudoaneurysm arising from this branch intrarenally (Fig. 3A) This vessel was then embolised using two $3 \times 2 \mathrm{~mm}$ fibered Gianturco coils (Fig. 3B). The patient made an uneventful recovery thereafter with no further haematuria. 


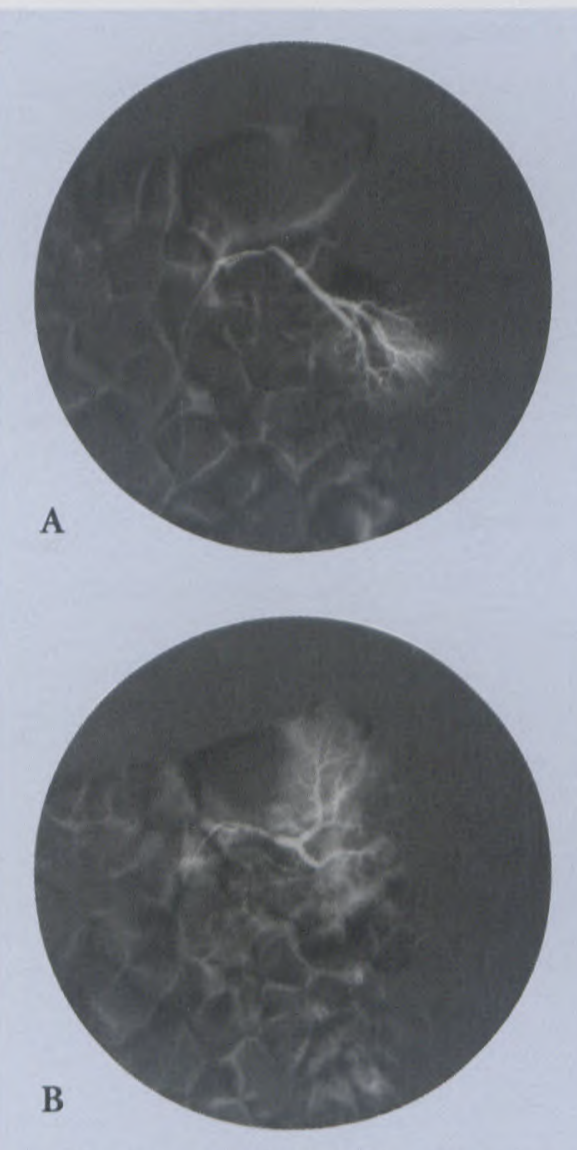

Fig. 2. Selective lower (2A) and upper (2B) multiple renal arteriograms.

Visual summation of the associated nephrograms led initially to an erroneous interpretation of a kidney possibly distorted by the perinephric haematoma or renal lacerations.

\section{Discussion}

Embryologically the definitive renal artery is not recognisable until the third month of fetal life. It arises from the most caudal of the three suprarenal arteries, all of which represent persistent mesonephric or lateral splanchnic arteries. Additional aberrant renal arteries thus represent persistent mesonephric arteries. ${ }^{2,3}$

Aberrant renal arteries can arise from the aorta anywhere between T11 and $\mathrm{L} 4$ and occur in approximately $25 \%$ of all individuals. ${ }^{4}$ They are commoner on the left than on the right. $^{1}$

There are two main types of multiple renal arteries.

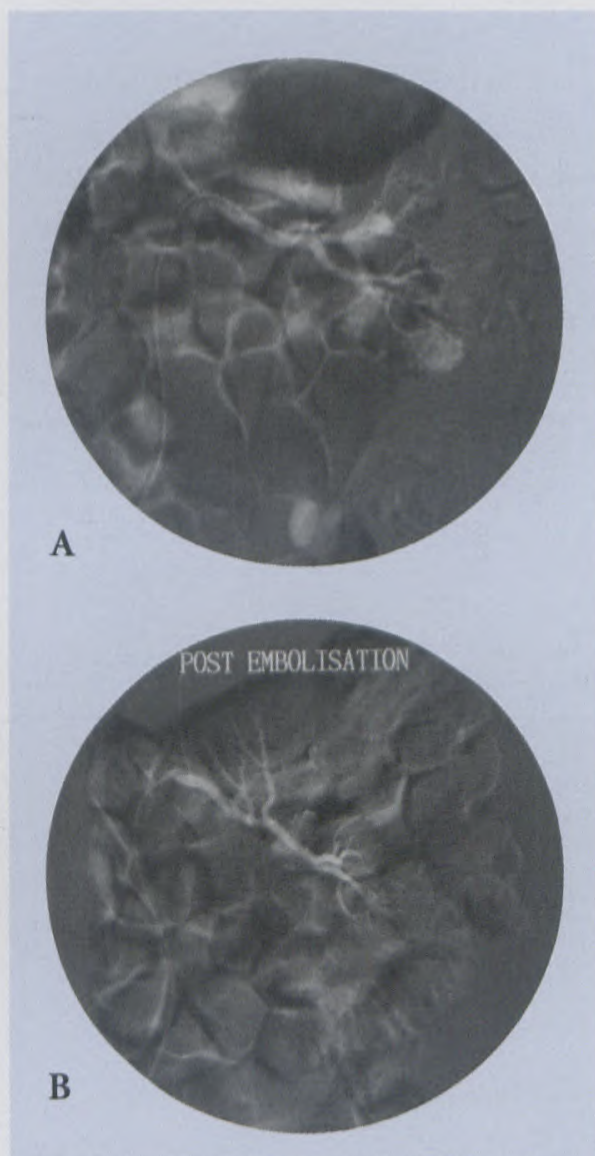

Fig. 3. A more careful search yielded a third renal artery harbouring a pseudoaneurysm and calyceal fistula related to one of its parenchymal branches (3A). The offending branch was then successfully occluded using two Gianturco coils (3B).

1. Polar arteries: Usually to the upper pole. These may arise from the main renal artery, from a hilar artery or directly from the aorta. They pass directly into the renal parenchyma without passing through the renal hilum.

2. Supplementary arteries: Usually to the lower pole and arising from the aorta near the origin of the main renal artery $(77 \%)$. These pass through the renal hilum into the parenchyma. They may occasionally also arise from other aortic branches including the iliac, superior and inferior mesenteric, coeliac, middle colic, lumbar or medial sacral arteries.

Further supply to small areas of the parenchyma can come from the capsular arteries as well. Each aberrant artery will supply its own area of the renal parenchyma, with little or no cross-supply between these separate compartments. Traditionally this anatomical information has been of great importance to the renal surgeon, particularly when planning renal transplant surgery. ${ }^{1}$ Here we have shown that the presence of these same anatomical variants can also provide a significant pitfall for the interventional radiologist in attempting to identify and eventually treat by endovascular means a lesion affecting the intrarenal arteries.

Several important technical issues have been raised by this case :

1. The quality of the images obtained during a digital subtraction arteriogram of the abdomen should always be optimised as much as possible, including sedation or full anaesthesia for restless patients, smooth muscle relaxants to paralyse the bowel and use of apnoea during the image acquisitions where possible.

2. An initial flush aortogram is essential and where possible should use a large enough field of view to include both nephrograms. The completeness of these nephrograms should be assessed at this point. If these are complete, then the nephrograms of selective renal arterial catheterisation of multiple renal arteries should, upon visually summating all their component parts, show a similar complete nephrogram.

3. In the face of overwhelming clinical evidence of a significant renal injury the study is not considered complete until the pathology is found or fully excluded, which may require a prolonged search for all accessory renal arteries present. 


\section{CASE REPORT}

In conclusion, our case demonstrates an important diagnostic pitfall associated with multiple renal arteries supplying a kidney. In the face of overwhelming clinical evidence of a major renal injury and two negative selective renal branch arteriograms of the same kidney, proper angiographic interpretation and persistence led to the discovery of a third vessel har- bouring a significant arterial injury. It is vital that in all similar circumstances the nephrograms are visually summated, and that all accessory renal arteries be thus identified and examined accordingly until a complete renal vascular anatomical study is achieved.

\section{References}

1. Pozniak MA, Balison DJ, Lee FT, Tambeaux RH, Vehling DT, Moon TD. CT angiography of potential renal transplant donors. Radiographics 1998; 18: 567-587.

2. Williams PL, Warwick R, eds. Gray's Anatomy. 36th ed. London: Churchill Livingstone, 1980: 212-213.

3. Carlson BM, ed. Pattens' Foundations of Embryology. 4th ed. New York: McGraw-Hill, 1981: 495-497.

4. Davidson AJ, Hartman DS, eds. Radiology of the Kidney and Urinary Tract. 2nd ed. Philadelphia: WB Saunders, 1994: 94. 